\title{
Directed Donation: Special Considerations and Review for Contemporary Clinical Practices
}

\author{
Gordon Wadge, MD, ${ }^{1}$ Jenny Zhang, MD, ${ }^{2}$ John Seal, MD, ${ }^{3,4}$ Edgar Shannon Cooper, MD, JD, ${ }^{5}$ Caroline \\ R. Alquist, $\mathrm{MD}, \mathrm{PhD}^{6}$
}

\begin{abstract}
${ }^{1}$ Department of Hematology and Oncology, Ochsner Clinic Foundation, New Orleans, LA 2 Department of Pathology, University of Arizona, Banner - University Medical Center Tucson, Tucson, AZ ${ }^{3}$ The University of Queensland Faculty of Medicine, Ochsner Clinical School, New Orleans, LA ${ }^{4}$ Section of Abdominal Transplant, Department of Transplant Surgery, Ochsner Clinic Foundation, New Orleans, LA ${ }^{5}$ Section of Transfusion Medicine and Histocompatibility, Department of Pathology and Laboratory Medicine, Ochsner Clinic Foundation, New Orleans, LA ${ }^{6}$ Hoxworth Blood Center Academic Unit and Department of Pathology, University of Cincinnati, Cincinnati, $\mathrm{OH}$
\end{abstract}

\begin{abstract}
Background: Directed blood donation is defined as the donation of blood or its components for the purpose of transfusion into a specified individual. Directed blood donation holds historic significance, and although practices as of 2021 encourage voluntary, nonrenumerated blood donations, public interest in directed donation remains. Requests to discuss the risks and benefits of directed donations are a common inquiry for transfusion medicine, transplant, and hematology/oncology professionals. This narrative review discusses the history of directed donation and summarizes directed donation considerations in the context of modern transfusion practices.

Methods: We conducted a systematic search of PubMed for published literature on the topic of directed blood donation and gathered information about its benefits and potential harms with respect to the variety of products used in transfusion medicine. Results: The drawbacks of directed donation include transfusion-transmitted infection risk, alloimmunization risk, increased transfusion-associated graft vs host disease risk, decreased expediency in treatment, and increased administrative burdens. However, a role remains for directed blood donation in specific patient populations, such as individuals with rare blood types or immunoglobulin A deficiencies, because of the difficulties in finding compatible blood for transfusion.

Conclusion: Clinicians should consider the risks and benefits when discussing directed blood donations with patients and family members.
\end{abstract}

Keywords: Blood component transfusion, blood donors, blood transfusion, directed donation

Address correspondence to Caroline R. Alquist, MD, PhD, Hoxworth Blood Center Academic Unit and Department of Pathology, University of Cincinnati, 3101 Highland Ave., 5th Floor TID, Cincinnati, OH 45237. Tel: (513) 558-1500. Email: caroline.alquist@uc.edu

\section{INTRODUCTION}

Directed blood donation is defined as the donation of blood or blood components for the purpose of transfusion into a specified individual, generally a recipient identified in advance of the collection. Directed blood donation has historic precedent. The first documented successful blood transfusion in 1818 was a directed donation from a husband to his postpartum wife. ${ }^{1}$ While this directed donation was born of necessity, transfusion practices as of 2021 encourage voluntary, nonrenumerated blood donations. ${ }^{2}$ However, public interest in directed donations remains, and requests to discuss the risks and benefits of directed donations are a common inquiry for transfusion medicine, transplant, and hematology/oncology professionals. This narrative review discusses the history of directed donation and summarizes directed donation considerations in the context of modern transfusion practices.
Autologous donation is outside the scope of this review, but discussions of autologous donations should include blood conservation techniques, intraoperative cell saver technology, historic transfusion requirements by procedure type, and the typical need for $>1$ unit when transfusion is required. ${ }^{3}$ Autologous donations are routinely used in lowrisk procedures, such as bone marrow donations, that historically require single-unit postoperative transfusions.

\section{METHODS}

We conducted a systematic search of PubMed for published literature on the topic of directed donations and also examined the reference lists of the articles. Topics searched included the history of transfusions, altruism, directed donations, hemophilia and other coagulopathies, and alloimmunization. We gathered information about the history of directed donations and the benefits and potential harms of 
directed donation with respect to the variety of products used in transfusion medicine.

\section{PSYCHOLOGY OF BLOOD DONATION}

In 1970, British social scientist Richard Titmuss published The Gift Relationship: From Human Blood to Social Policy. This book greatly influenced both British and US transfusion practices by asserting that blood donation must occur as an altruistic act to avoid human exploitation, commercialism, and risk. ${ }^{4,5}$ Titmuss warned that paid donor arrangements could result in wasteful and inefficient donation systems with exploitative redistribution of blood products. He predicted that a nonaltruistic path would contribute to the degradation of society via enhanced selfish interests and personal gain..$^{4,5}$

With respect to blood donation, 2 forms of altruism must be considered: induced altruism and kin altruism. Induced altruism is defined as giving without expected reciprocal benefit. ${ }^{4,5}$ Voluntary, nonrenumerated blood donations are an example of induced altruism. Donated products enter the anonymized pool of available blood without reference to their eventual recipient. Kin altruism is defined as increased altruistic action if the person aided is known to the giver. ${ }^{4,5}$ Kin altruism is uniquely satisfied by directed donation and is a driving influence on directed donation behavior. In general, studies identify altruism as the primary reason for modern blood donations. ${ }^{6}$ Other motivating factors behind the decision to donate include gratitude, reciprocity, replacement, duty, having a rare blood group, benefit (eg, discovery of blood type), and personal appeal. ${ }^{4,5}$

Directed donations may be influenced by extrinsic pressures, such as coercion or monetary compensation, particularly in times of crisis. ${ }^{2}$ Ramifications of such pressure may include inaccurate answers on predonation screening questions. The US Food and Drug Administration (FDA)approved Uniform Donor History Questionnaire (UDHQ) standardizes blood donor screenings. ${ }^{6}$ Questions about high-risk behaviors of the donor include male-to-male sex and intravenous drug use. Reports have shown, however, that donors have sometimes answered untruthfully because of donor perception of their own blood safety, their faith in the fidelity of infectious disease testing, confidentiality concerns, and their altruistic desire to save lives. ${ }^{7,8}$ UDHQ highrisk behaviors are correlated with transfusion-transmissible diseases such as hepatitis and HIV. In the case of a directed donation, donors may be pressured to answer the screening questions untruthfully because they are being relied upon by the intended recipients.

Additionally, infectious disease testing is not foolproof. Testing modalities combine serology testing with nucleic acid technology testing, but infectious disease testing is not comprehensive, particularly for viruses with controversial transfusion-transmission risk, such as Epstein-Barr virus, and for emerging viral infections. ${ }^{9}$ False negatives can also occur, particularly during acute infection periods when hostresponse serologic markers have not yet developed. .,10 $^{10}$ Thus, while the risk of transmission per unit transfused for viruses such as HIV and hepatitis $B$ virus (HBV) is extremely low (1 in 1 million or lower for each), the risk is not zero, and further testing has been posited as ineffectual in maximally lowering the risk. ${ }^{9,10}$ The enhanced infectious disease risk of directed donation is further discussed in a subsequent section. While incorrect answers on the UDHQ are a poten- tial issue for all donations, directed donations motivated by kin altruism may increase the risk of a donor answering the UDHQ untruthfully.

\section{HISTORIC NEED FOR DIRECTED DONATION}

Historically, directed donation served to minimize donor exposure in frequently transfused patients. Patients receiving the bulk of frequent plasma and plasma-derivative transfusions include those with hemophilia and von Willebrand disease. Prior to treatments such as recombinant replacement factors and desmopressin, whole blood or plasma were the only available treatments for a bleeding episode, the frequency of which varied based on disease severity. ${ }^{10}$

Hereditary hemophilia is a set of X-linked disorders characterized by a lack of factor 8 in hemophilia A or factor 9 in hemophilia B. Frequent transfusions of plasma-derived products such as cryoprecipitate and plasma factor concentrates became synonymous with inadvertent transmission of viral-borne illnesses to the hemophilia population, including HIV, HBV, and hepatitis C (HCV). Among US hemophiliacs treated between 1982 and 1984, 74\% of patients who received factor 8 concentrates and up to $90 \%$ who received factor 9 concentrates were positive for antibodies to HIV. ${ }^{11}$ Cirrhosis, likely secondary to hepatitis transmission, was also seen in $15 \%$ to $38 \%$ of patients with hemophilia. ${ }^{12}$ These infection rates may be attributed to the amplified risk of multiple donor exposures, paid plasma donation sources using nonaltruistic donors, and the lack of screening for HIV risk factors at that time. ${ }^{13}$ Effective recombinant replacement factors were not developed until the late 1980s, and patients with hemophilia no longer had to receive as many plasma and plasma-derivative transfusions, decreasing their exposure to transfusion-transmitted infections. ${ }^{12}$

Von Willebrand disease is secondary to a qualitative or quantitative defect in the von Willebrand protein, which is vital to platelet plug formation and prevention of the inactivation and clearance of factor 8 . One of the first treatments for von Willebrand disease was cryoprecipitate derived from human plasma in $1964 .{ }^{14}$ As for patients with hemophilia, patients with von Willebrand disease also had a risk of acquiring viral-borne illnesses such as HIV, HBV, and HCV, although they did not require transfusions as frequently. ${ }^{15}$ Desmopressin was not found to be clinically useful in patients with type 1 von Willebrand disease, the most prevalent form, until 1977. ${ }^{14,16}$ As a result, treatment no longer relied on frequent cryoprecipitate transfusions, decreasing transfusion-transmitted infection risks. In 1981, Humate-P, a pasteurized formulation of factor 8 and von Willebrand factor, was developed to treat types 2 and 3 von Willebrand disease. ${ }^{17}$

Modern recombinant products and improved processing technology have greatly decreased infectious disease risk for patients with hemophilia and von Willebrand disease.

\section{TRANSFUSION-RELATED INFECTIOUS DISEASE RISK}

Interest in directed donation, particularly from a friend or an acquaintance, is often linked to a misperception of increased safety. Directed donation inquiries reached an all-time high in the 1980s around the discovery of HIV and its blood-borne transmission. ${ }^{18}$ Requests for directed 
donations have since decreased as laboratory evaluation and transfusion have become safer. ${ }^{18}$ The FDA mandates that all US blood products intended for transfusion be tested for HBV, HCV, HIV-1, human T-lymphotropic virus (HTLV) types 1 and 2, Trypanosoma cruzi parasites, and Zika virus. The rate of contracting HIV from a transfusion is currently estimated to be 1 in 1.5 million, while transmission rate estimates for HBV and HCV are 1 in 1 million for HBV and 1 in 1.2 million for HCV. ${ }^{19,20}$

Despite improvements in infectious disease screening and testing, however, directed donations are associated with an increased risk of transfusion-transmitted infection compared to blood from anonymous volunteer donors. ${ }^{18}$ One retrospective study found reactive rates per 100,000 donations for volunteer vs directed donors as follows: HIV (2.9 vs 7.2), HBV (12.4 vs 40.1), HCV (32.2 vs 93), and HTLV (2.5 vs 18.6). The crude odds ratios for HIV, HBV, and HTLV transmission from directed donor blood products are significantly higher than those seen with volunteer donors. ${ }^{18}$ The increased risk of a transfusion-transmitted infection in directed donors remains even when the donor is a parent of the patient. Combined rates of HIV, HBV, HCV, HTLV, and syphilis reactivity in parental directed donations were $6.33 \%$ vs $1.3 \%$ for community donations. ${ }^{21}$ Thus, increased risk of transfusion-transmitted infections in directed donor blood products should be considered because the detection of such infections would preclude the donated blood products from use.

\section{ALLOIMMUNIZATION RISKS}

All transfusions carry an alloimmunization risk to human leukocyte antigens (HLAs) that may impact future transfusion and transplant outcomes. The immune system uses cellsurface HLAs and antibodies to identify and destroy non-self cells. Exposure to non-self HLAs on transfusion-transmitted white blood cells may result in a host response of alloantibody formation to those non-self HLAs. More than $30 \%$ of patients requiring multiple transfusions will develop alloantibodies, although this rate may be lower in patients with compromised immune systems. ${ }^{22}$ The risk of developing alloimmunization after red blood cell transfusion is estimated to be $0.5 \%$ per unit transfused or $1.15 \%$ per transfusion episode. ${ }^{22}$ A risk of alloimmunization secondary to residual leukocytes exists with plasma transfusions. However, the process of removing leukocytes through filtration from a blood product before transfusion, known as leukoreduction, has been shown to mitigate alloimmunization risk. ${ }^{23}$ Alloimmunization may also occur secondary to platelet transfusions. In one study, alloimmunization occurred in $45 \%$ of non-leukocyte-reduced platelet transfusions. ${ }^{24}$ Even following leukoreduction, $18 \%$ of platelet transfusion recipients may become alloimmunized. ${ }^{24}$

Anti-HLA antibody formation to transfused blood products has implications for a patient's future response to transfusions and for potential solid organ and bone marrow transplant recipients. Patients who develop anti-HLA antibodies may no longer appropriately respond to platelet transfusions that carry the relevant HLAs on their cell surface. Additionally, the presence of anti-HLA antibodies may rule out potential bone marrow and solid organ donors. Alloimmunization can have deleterious effects on solid organ and bone marrow transplants, including early death, impaired engraftment/organ tolerance, and increased rejection. ${ }^{25-27}$

The risk of alloimmunization becomes particularly important to consider for family members asking to directly donate blood products to a patient. Family members are the most common donor sources for bone marrow and solid organ donation given the likelihood of patient blood type and HLA matching. The development of anti-HLA antibodies following a transfusion from a family member's directed donation may eliminate that family member and relatives with similar typing as donor sources for future bone marrow or organ transplants.

\section{TRANSFUSION-ASSOCIATED GRAFT VS HOST DISEASE}

Transfusion-associated graft vs host disease (TA-GVHD) is the failure of a recipient's immune system to recognize donor lymphocytes in a transfused product as foreign, allowing donor cells to mount a destructive response against the recipient's lymphoid tissue. Typically manifesting within 30 days after transfusion, TA-GVHD symptoms include fever, nausea, vomiting, mucositis, diarrhea, gastrointestinal bleeding, skin lesions, hepatic dysfunction, and pancytopenia. Without any known effective treatments beyond supportive care, mortality from TA-GVHD is $>90 \% .^{28}$

TA-GVHD most commonly occurs following blood donation from a related donor with an HLA profile similar to the recipient's and in populations with limited HLA diversity. ${ }^{28}$ Leukoreduction alone is not sufficient to prevent TA-GVHD. To prevent TA-GVHD, blood products from relatives and those with predictably similar HLA profiles undergo gamma irradiation. This treatment effectively damages lymphocyte DNA to prevent posttransfusion engraftment and proliferation capabilities. ${ }^{28}$ In Japan, where the first case of TAGVHD was reported in 1955, TA-GVHD incidence dropped from $0.15 \%$ between 1981 and 1986 to 0 cases in 2000 and 2001 following implementation of measures such as irradiation. ${ }^{28,29}$

Most directed donations are subject to irradiation regardless of recipient relation, but TA-GVHD remains a theoretical concern with any cellular product transfusion. In some areas, access to an irradiator may not be available, which either precludes the use of blood products from those with similar HLA profiles or, if the blood products are used, increases the risk of TA-GVHD occurring. Irradiated units also raise concerns for elevated plasma potassium levels because of the hemolysis of red blood cells. ${ }^{30}$ Hyperkalemia poses a potential risk for certain populations such as neonates and patients with renal failure and should be taken into consideration.

\section{TESTING RESTRAINTS AND ADMINISTRATION DIFFICULTIES}

As stated previously, the FDA mandates that all US blood products be tested for HBV, HCV, HIV-1, HTLV types 1 and 2, Trypanosoma cruzi parasites, and Zika virus. ${ }^{19,20}$ The turnaround time for this required testing is often overlooked when planning for directed donations. Directed donations are not immediately available for transfusion; their release is contingent upon testing completion and acceptable test results. Depending on the testing and product preparation 
process of the particular blood bank, the period from collection to product availability for transfusion may be $>48$ hours. Because of the turnaround time, directed donation is a less feasible option than the use of voluntary, nonrenumerated blood donations when a transfusion is needed expediently because the latter products would have already been tested and prepared for transfusion.

In addition, directed donations have increased administrative requirements. Facility-specific circumstances may require that blood products be donated and collected at a separate facility than where the patient receives the transfusion. In comparison, a facility would already have blood products from voluntary, nonrenumerated blood donations in stock. Extra care must be taken to ensure the directed donation units are appropriately labeled, tested, and crossmatched for the intended patient, transported to the patient's specific location, and transfused to the correct patient. ${ }^{30,31}$ Directed donations therefore involve administrative burdens as well as time constraints.

\section{MODERN APPLICATIONS OF DIRECTED DONATION}

Directed donation continues to play an important, albeit limited, role in transfusion medicine. Individuals requiring HLA-matched platelets, granulocytes, and products for immunoglobulin A ( $\lg A)$ deficiency and individuals with fetal and neonatal alloimmune thrombocytopenia or rare blood types all rely on directed donors. ${ }^{31}$

Patients with rare blood types and significant blood antigen alloimmunization rely on donors of compatible blood. In 1998, the American Rare Donor Program (ARDP) was developed and tasked with keeping a registry of rare blood donor information. Managed by the American Red Cross National Reference Laboratory for Blood Group Serology in conjunction with the AABB (formerly the American Association of Blood Banks), the function of the ARDP is to facilitate donor identification for patients with rare blood types and individuals with antibodies to high-frequency antigens or with multiple common antibodies. ${ }^{32}$ Rare blood types are those that match $<1$ in 1,000 donations. ${ }^{32}$ Very rare products are those identified in $<1$ in 10,000 donated units. Examples of such phenotypes include the Bombay (found in 1 in 2,000 to 1 in 13,000 donations) and Indian (found in 1 in 250,000 donations) phenotypes. ${ }^{33}$ Donor information is updated semiannually in the ARDP. When a rare or very rare blood type is requested, the ARDP requests testing of a patient's sibling(s) or initiates recruitment of registered rare donors for directed donation. ${ }^{32}$

The ARDP services for donor identification are also commonly used by patients with sickle cell disease who have a high propensity for developing alloantibodies. From January 2005 to June 2006, 33\% of requests for red blood cell products were for alloimmunized patients with sickle cell disease. ${ }^{34}$ High alloimmunization rates $(7 \%$ to $47 \%)$ in the United States have in part been attributed to the fact that the majority of blood donors in the United States are of European descent. ${ }^{35}$ Europeans have different allele frequencies than most patients with sickle cell disease. For example, $\mathrm{C}$ and $\mathrm{E}$ of the Rhesus (Rh) antigen group, K of the Kell (KEL) antigen group, Fy(a) of the Duffy (Fy) antigen group, Jk(b) of the Kidd (Jk) antigen group, and S of the MNS antigen group are more frequently encountered in persons of European decent than in persons of African descent. ${ }^{35}$ The ARDP plays a crucial role in matching patients with rare blood types or significant alloimmunizations with specific donors for directed donation.

Alloimmunization is a significant cause of platelet refractoriness. Patients are defined as platelet refractory when their platelet counts fail to respond appropriately to 2 separate platelet transfusions. Platelet refractoriness has immune and nonimmune causes. Immune causes include human platelet antigen (HPA), or more commonly, HLA antibodies. As previously mentioned, leukoreduction may decrease HLA alloimmunization risk, but the risk varies with underlying diagnosis, history of pregnancy, and transfusion exposures. ${ }^{33,36}$ Nonimmune causes of platelet refractoriness include sepsis, splenomegaly, disseminated intravascular coagulation, and medications. ${ }^{37}$

Once nonimmune causes are excluded, a platelet antibody screen should be performed to evaluate plateletrefractory patients. If the screen indicates an alloimmune cause, HLA-appropriate products can be identified via cross-matching of available ABO-identical platelet inventory or by seeking an HLA-compatible donor. To identify such donors, HLA typing of the recipient and potential donors and identification of preexisting recipient HLA antibodies must be performed. An appropriate platelet donor should have HLA-A and HLA-B types in common with the recipient, or at a minimum, lack the HLAs to which the recipient has produced antibodies. ${ }^{38}$ HLA-matched platelets can be difficult and expensive to obtain. ${ }^{38}$ HLA-typed donor databases are therefore crucial to the identification of altruistic donors who are willing to provide compatible products to a patient in times of need. Thus, for patients with rare blood types or alloimmunization, a directed donation would serve them better than the voluntary, nonrenumerated blood donations typically available at a blood bank.

A form of directed donation in current use is granulocyte transfusion. While their value and efficacy are controversial, granulocyte transfusions may be given prophylactically to patients with chronic granulomatous disease or with severe neutropenia from a prolonged infection that has failed multiple antibiotics. ${ }^{39}$ Transfusions provide patients time to mount their own responses to the infection. ${ }^{40}$ While most centers use family members as donors, volunteer donors may also come from the community to provide ABO-compatible granulocyte products. ${ }^{41}$ Prior to collection, donors are administered mobilization medications such as steroids and/or granulocyte colony-stimulating factor to increase circulating neutrophils in the peripheral blood. ${ }^{41}$ Directed donation of granulocyte products is not without risk to both donor and recipient. Donor mobilization medications have potential risks of thrombosis, splenic rupture, and anaphylactoid reactions. Risks to the recipient are a $10 \%$ rate of alloimmunization and a host of potential postinfusion reactions, including fever, chills, rash, shortness of breath, pulmonary distress, and cytomegalovirus infections. ${ }^{42,43}$ Once granulocyte transfusions are initiated, irradiated granulocyte infusions are usually given daily and require recruitment of multiple individual donors. This treatment modality hinges on the availability of willing and available donors because of the short, 24-hour storage life 
of granulocytes, making a directed donation more feasible than voluntary, nonrenumerated blood donation.

IgA-deficient patients may require directed donations because they can develop anti-lgA antibodies that result in an anaphylactic reaction to all plasma-containing blood products. As such, IgA-deficient patients require washing of cellular blood products, which replaces the blood product plasma with normal saline and in the process removes microaggregates, leukocytes, and plasma proteins, including IgA. If a patient is receiving plasma derivatives, the products must come from an IgA-deficient donor. IgA-deficient products can be ordered from blood product distributors who can contact willing prescreened donors.

Fetal and neonatal alloimmune thrombocytopenia is a relatively rare condition that occurs when a mother develops antibodies against the paternal (non-self) HPAs on fetal platelets. ${ }^{44}$ Outcomes of this condition may include fetal intracranial hemorrhage and bleeding diatheses. Initial treatments of this condition-first performed in 1983-required directed donation of irradiated and washed platelets from the mother to the developing fetus through intrauterine transfusions. ${ }^{44-46}$ Current treatment of fetal alloimmune thrombocytopenia involves maternally administered intravenous immunoglobulin with or without steroids. ${ }^{44}$ Intrauterine platelet transfusions, maternally derived or special ordered, are now reserved as a second-line treatment. After birth, the primary treatment for neonatal alloimmune thrombocytopenia is platelet transfusion. The best platelets for transfusion are those from HPA-1bb/5aa-typed platelet donors who are contacted for donation. Newborns may also receive readily available random platelets or irradiated and washed maternal platelets with similar outcomes. ${ }^{44}$

\section{CONCLUSION}

Inquiries about directed blood donation are common in clinical practice and present unique challenges. While induced altruism is the most common motivating factor for blood donations, the kin altruism behind directed donations introduces potential confounding motivations of gratitude, reciprocity, duty, and personal gain that can ultimately compromise the safety of the donation process. Modern practice favors the collection of blood products from voluntary nonremunerated donations because of the risks present in blood donations given for reasons other than altruism. However, improvements in blood product screening reduce that risk considerably, and specific risks associated with directed donation should be carefully considered. Providers and patients should be advised of directed donation drawbacks, including transfusion-transmitted infection, alloimmunization risk, increased TA-GVHD risk, decreased expediency in treatment, and increased administrative burdens.

While many requests for directed donations are rooted in historic concerns, a role remains for directed donations in practice. The best or only option for patients with rare blood types, IgA deficiency, or significant alloimmunization may be a directed donor.

\section{ACKNOWLEDGMENTS}

The authors have no financial or proprietary interest in the subject matter of this article.

\section{REFERENCES}

1. Blundell J. Some account of a case of obstinate vomiting, in which an attempt was made to prolong life by injection of blood into the veins. Med Chir Trans. 1819;10(Pt 2):295-311.

2. WHO Expert Group. Expert consensus statement on achieving self-sufficiency in safe blood and blood products, based on voluntary non-remunerated blood donation (VNRBD). Vox Sang. 2012;103(4):337-342. doi: 10.1111/j.1423-0410.2012.01630.x

3. Klein HG, McCurdy PR, Nemo G, Rogus SD, Sloand E, Fink BJ; National Heart, Lung, and Blood Institute Expert Panel on the use of Autologous Blood. Transfusion alert: use of autologous blood. Transfusion. 1995;35(8):703-711. doi: 10.1046/j.1537-2995.1995.35895357904.x

4. Titmuss RM. The Gift Relationship: From Human Blood to Social Policy. Geroge Allen and Unwin; 1970.

5. Rapport FL, Maggs CJ. Titmuss and the gift relationship: altruism revisited. J Adv Nurs. 2002;40(5):495-503. doi: 10.1046/j.1365-2648.2002.02406.x

6. Fridey JL, Townsend MJ, Kessler DA, Gregory KR. A question of clarity: redesigning the American Association of Blood Banks blood donor history questionnaire-a chronology and model for donor screening. Transfus Med Rev. 2007;21(3):181-204. doi: 10.1016/j.tmrv.2007.03.001

7. Hughes S, Sheon N, Siedle-Khan B, Custer B. Saving lives, maintaining safety, and science-based policy: qualitative interview findings from the Blood Donation Rules Opinion Study (Blood DROPS). Transfusion. 2015;55(12):2835-2841. doi: $10.1111 /$ trf.13268

8. Grenfell P, Nutland W, McManus S, Datta J, Soldan K, Wellings $\mathrm{K}$. Views and experiences of men who have sex with men on the ban on blood donation: a cross sectional survey with qualitative interviews. BMJ. 2011;343:d5604. doi: $10.1136 / \mathrm{bmj} . d 5604$

9. Busch MP, Bloch EM, Kleinman S. Prevention of transfusion-transmitted infections. Blood. 2019;133(17):1854-1864. doi: 10.1182/blood-2018-11-833996

10. Franchini M, Mannucci PM. Past, present and future of hemophilia: a narrative review. Orphanet J Rare Dis. 2012;7:24. doi: 10.1186/1750-1172-7-24

11. Goedert JJ, Sarngadharan MG, Eyster ME, et al. Antibodies reactive with human $T$ cell leukemia viruses in the serum of hemophiliacs receiving factor VIII concentrate. Blood. 1985;65(2):492-495.

12. Rosendaal FR, Smit C, Briët E. Hemophilia treatment in historical perspective: a review of medical and social developments. Ann Hematol. 1991;62(1):5-15. doi: 10.1007/bf01714977

13. Fletcher ML, Trowell JM, Craske J, Pavier K, Rizza CR. Non-A non-B hepatitis after transfusion of factor VIII in infrequently treated patients. BrMed J (Clin Res Ed). 1983;287(6407):1754-1757. doi: 10.1136/bmj.287.6407.1754

14. Federici $A B$, Berntorp $E$, Lee CA. The 80th anniversary of von Willebrand's disease: history, management and research. Haemophilia. 2006;12(6):563-572. doi: 10.1111/j.1365-2516.2006.01393.x

15. Federici AB, Santagostino E, Rumi MG, et al. The natural history of hepatitis $C$ virus infection in Italian patients with von Willebrand's disease: a cohort study. Haematologica. 2006;91(4):503-508.

16. Sharma R, Flood VH. Advances in the diagnosis and treatment of Von Willebrand disease. Blood. 2017;130(22):2386-2391. doi: 10.1182/blood-2017-05-782029

17. Mannucci PM. Treatment of von Willebrand's disease. N EnglJ Med. 2004;12;351(7):683-694. doi: 10.1056/NEJMra040403 
18. Dorsey KA, Moritz ED, Steele WR, Eder AF, Stramer SL. A comparison of human immunodeficiency virus, hepatitis $C$ virus, hepatitis $B$ virus, and human T-lymphotropic virus marker rates for directed versus volunteer blood donations to the American Red Cross during 2005 to 2010. Transfusion. 2013;53(6):1250-1256. doi: 10.1111/j.1537-2995.2012.03904.x

19. Stramer SL, Notari EP, Krysztof DE, Dodd RY. Hepatitis B virus testing by minipool nucleic acid testing: does it improve blood safety? Transfusion. 2013;53(10 Pt 2):2449-2458. doi: $10.1111 /$ trf.12213

20. Zou S, Stramer SL, Dodd RY. Donor testing and risk: current prevalence, incidence, and residual risk of transfusion-transmissible agents in US allogeneic donations. Transfus Med Rev. 2012;26(2):119-128. doi: 10.1016/j.tmrv.2011.07.007

21. Jacquot C, Seo A, Miller PM, et al. Parental versus non-parental-directed donation: an 11-year experience of infectious disease testing at a pediatric tertiary care blood donor center. Transfusion. 2017;57(11):2799-2803. doi: $10.1111 /$ trf.14312

22. Schonewille $H$, Haak HL, van Zijl AM. Alloimmunization after blood transfusion in patients with hematologic and oncologic diseases. Transfusion. 1999;39(7):763-771. doi: 10.1046/j.1537-2995.1999.39070763.x

23. Hiruma K, Okuyama Y. Effect of leucocyte reduction on the potential alloimmunogenicity of leucocytes in fresh-frozen plasma products. Vox Sang. 2001;80(1):51-56. doi: 10.1046/j.1423-0410.2001.00011.x

24. Trial to Reduce Alloimmunization to Platelets Study Group. Leukocyte reduction and ultraviolet $B$ irradiation of platelets to prevent alloimmunization and refractoriness to platelet transfusions. N Engl J Med. 1997;337(26):1861-1869. doi: 10.1056/NEJM199712253372601

25. Lerut E, Van Damme B, Noizat-Pirenne F, et al. Duffy and Kidd blood group antigens: minor histocompatibility antigens involved in renal allograft rejection? Transfusion. 2007;47(1):28-40. doi: 10.1111/j.1537-2995.2007.01060.x

26. Au WY, Liu CL, Lo CM, Fan ST, Lam MF, Lam CK. Red blood cell alloantibodies and liver transplantation in Chinese patients. Transplantation. 2003;75(11):1904-1906. doi: 10.1097/01.TP.0000065741.31599.9C

27. Makroo RN, Agrawal S, Chowdhry M, Bhatia A, Thakur UK. Red cell alloimmunization \& role of advanced immunohaematological support in liver transplantation. Indian J Med Res. 2017;145(4):488-491.

28. Schroeder ML. Transfusion-associated graft-versus-host disease. Br J Haematol. 2002;117(2):275-287. doi: 10.1046/j.1365-2141.2002.03450.x

29. Shimoda T. On postoperative erythroderma. Surgery. Article in Japanese. 1955;17:487-492. (in Japanese).

30. Wylie B. Which methods of donor recruitment give the safest donors? Malays J Pathol. 1993;15(2):99-103.

31. Chaffin J. Blood Bank Guy Essentials Podcast. 062CE: Autologous and Directed Donation with Julie Karp. January 18, 2019. Accessed February 4, 2021. bbguy.org/2019/01/18/062
32. Flickinger C, Petrone T, Church A. Review: American rare donor program. Immunohematology. 2004;20(4):239-243.

33. Dipta TF, Hossain AZ. The Bombay blood group: are we out of risk? Mymensingh Med J. 2011;20(3):536-540.

34. Flickinger $C$. In search of red blood cells for alloimmunized patients with sickle cell disease. Immunohematology. 2006;22(3):136-142.

35. Yazdanbakhsh K, Ware RE, Noizat-Pirenne F. Red blood cell alloimmunization in sickle cell disease: pathophysiology, risk factors, and transfusion management. Blood. 2019;120(3):528-537. doi: 10.1182/blood-2011-11-327361

36. Nelson KA, Aldea GS, Warner $P$, et al. Transfusion-related immunomodulation: gamma irradiation alters the effects of leukoreduction on alloimmunization. Transfusion. 2019;59(11):3396-3404. doi: 10.1111/trf.15555

37. Hod E, Schwartz J. Platelet transfusion refractoriness. Br J Haematol. 2008;142(3):348-360. doi: 10.1111/j.1365-2141.2008.07189.x

38. Pavenski K, Freedman J, Semple JW. HLA alloimmunization against platelet transfusions: pathophysiology, significance, prevention and management. Tissue Antigens. 2012;79(4):237-245. doi: 10.1111/j.1399-0039.2012.01852.x

39. West KA, Conry-Cantilena C. Granulocyte transfusions: current science and perspectives. Semin Hematol. 2019;56(4):241-247. doi: 10.1053/j.seminhematol.2019.11.002

40. Gea-Banacloche J. Granulocyte transfusions: a concise review for practitioners. Cytotherapy. 2017;19(11):1256-1269. doi: 10.1016/j.jcyt.2017.08.012

41. Hübel K, Carter RA, Liles WC, et al. Granulocyte transfusion therapy for infections in candidates and recipients of HPC transplantation: a comparative analysis of feasibility and outcome for community donors versus related donors. Transfusion. 2002;42(11):1414-1421. doi: 10.1046/j.1537-2995.2002.00249.x

42. Massey E, Harding K, Kahan BC, et al. The granulocytes in neutropenia 1 (GIN 1) study: a safety study of granulocytes collected from whole blood and stored in additive solution and plasma. Transfus Med. 2012;22(4):277-284. doi: 10.1111/j.1365-3148.2012.01152.x

43. Price TH, Boeckh M, Harrison RW, et al. Efficacy of transfusion with granulocytes from G-CSF/dexamethasone-treated donors in neutropenic patients with infection. Blood. 2015;126(18):2153-2161. doi: 10.1182/blood-2015-05-645986

44. Winkelhorst D, Oepkes D, Lopriore E. Fetal and neonatal alloimmune thrombocytopenia: evidence based antenatal and postnatal management strategies. Expert Rev Hematol. 2017;10(8):729-737. doi: 10.1080/17474086.2017.1346471

45. Reznikoff-Etievant MF. Management of alloimmune neonatal and antenatal thrombocytopenia. Vox Sang. 1988;55(4):193-201. doi: 10.1111/j.1423-0410.1988.tb04697.x

46. Daffos F, Capella-Pavlovsky M, Forestier F. Fetal blood sampling via the umbilical cord using a needle guided by ultrasound. Report of 66 cases. Prenat Diagn. 1983;3(4):271-277. doi: $10.1002 /$ pd. 1970030402

This article meets the Accreditation Council for Graduate Medical Education and the American Board of Medical Specialties Maintenance of Certification competencies for Patient Care and Medical Knowledge.

(C2021 by the author(s); licensee Ochsner Journal, Ochsner Clinic Foundation, New Orleans, LA. This article is an open (c) (i) access article distributed under the terms and conditions of the Creative Commons Attribution (CC BY) license (creativecommons.org/licenses/by/4.0/legalcode) that permits unrestricted use, distribution, and reproduction in any medium, provided the original author(s) and source are credited. 